\title{
A rare presentation of splenic rupture
}

\author{
Trevor J. GibBS \\ M.B., Ch.B. \\ Robert A. Sells* \\ F.R.C.S.
}

\author{
Alastair J. Bellingham \\ F.R.C.P.
}

\author{
Department of Haematology, University of Liverpool, and \\ *Renal Transplant Unit, Liverpool Royal Infirmary, Liverpool
}

\begin{abstract}
Summary
Traumatic rupture of the spleen is a well known problem, complicating many types of abdominal injury. In contrast, a spontaneous rupture is a rare occurrence, mainly associated with a large spleen resulting from infections, the most common in this country being infectious mononucleosis. It is also a rare complication of leukaemias and lymphomas and the case presented here demonstrates a peculiar diagnostic problem.
\end{abstract}

\section{Case report}

First admission

A 28-year-old journalist presented to this hospital in October 1973 with symptoms of anaemia. There was no history of a bleeding diathesis, infection or significant past or family history.

Examination showed a thin man, anaemic, not icteric, with a liver palpable $5 \mathrm{~cm}$ below the right costal margin and a spleen $11 \mathrm{~cm}$ below the left costal margin. There was no lymphadenopathy. There were two small retinal haemorrhages present, one in each eye.

Investigations. $\mathrm{Hb} 7.0 \mathrm{~g} / \mathrm{dl}$, WBC $5.5 \times 10^{9} / 1$, platelets $80 \times 10^{9} / 1$, differential WBC: $16 \%$ neutrophils, $20 \%$ lymphocytes, $2 \%$ monocytes and $62 \%$ lymphoblasts. The marrow was completely replaced with lymphoblasts, consistent with a diagnosis of acute lymphoblastic leukaemia. Blood urea was $7 \cdot 5$ $\mathrm{mmol} / \mathrm{l}$, uric acid $0.20 \mathrm{mmol} / \mathrm{l}$, serum proteins, plasma electrolytes and liver function tests were all normal. There was no lymphadenopathy visible on chest X-ray.

Treatment. He was treated with standard induction of vincristine and prednisolone. After 12 weeks the liver and spleen were no longer palpable, remission was shown by a normal peripheral blood and the marrow aspirate which contained less than $5 \%$

Correspondence: Dr T. J. Gibbs, Department of Haematology, University of Liverpool, Crown Street, P.O. Box 147, Liverpool L69 3BX. blast cells. Cranio-spinal irradiation together with intrathecal methotrexate was given for CNS prophylaxis. Following this he then received two courses of cyclical remission chemotherapy each of 12 weeks' duration utilizing methotrexate, prednisolone, adriamycin, cytosine, 6-mercaptopurine and cyclophosphamide. He remained in remission and at full time work with a normal peripheral blood count except for an occasional suppression of the white cell count necessitating temporary reduction in dosage.

\section{Second admission}

In March 1975 while receiving his third cycle of remission chemotherapy he became anaemic and thrombocytopenic and was readmitted.

Investigations. $\mathrm{Hb} 8.6 \mathrm{~g} / \mathrm{dl}$, WBC $3.3 \times 10^{9} / 1$, platelets $71 \times 10^{9} / 1$, with an occasional primitive cell seen in the peripheral blood. Marrow examination confirmed that he was in relapse.

Treatment. Re-induction with vincristine and prednisolone was begun, but although there was a slight improvement in the peripheral blood and marrow after 6 weeks, remission had not been achieved. A further attempt at re-induction was made in April 1975 with a combination of cytosine and daunorubicin.

\section{Third admission}

It was necessary to readmit him for a third time because of symptoms of postural hypotension with a feeling of upper abdominal fullness and discomfort, especially in the left hypochondrium.

Examination. He was found to be anaemic and his blood pressure fell from $16 \cdot 6 / 10.6 \mathrm{kPa}$ lying to $11 \cdot 3 / 9 \cdot 3 \mathrm{kPa}$ standing. He had a $3-\mathrm{cm}$ hepatomegaly and a $5-\mathrm{cm}$ tender splenomegaly but no ascites and his bowel sounds were normal. Although there was no frank bleeding, he had numerous skin petechiae.

Investigations. $\mathrm{Hb} 9.0 \mathrm{~g} / \mathrm{dl}$, WBC $47 \times 10^{9} / 1(37 \%$ blasts) and platelets $38 \times 10^{9} / 1$.

Treatment. His symptoms were attributed to his 
anaemia and the rapid enlargement of his spleen. He was treated with transfusion and further chemotherapy with some benefit, his spleen size regressing.

On the fifth day of chemotherapy, whilst lying in bed, he complained of a sudden onset of upper abdominal pain and nausea. He did not admit to any shoulder-tip pain. Examination showed a shocked patient, pale with cold, clammy peripheries, a weak rapid pulse and blood pressure was $12 \cdot 0 / 9 \cdot 3 \mathrm{kPa}$. There was upper abdominal distension so marked that the spleen was no longer palpable. There was diffuse tenderness but no guarding, rigidity or rebound tenderness. Bowel sounds were normal and there was no melaena on rectal examination. X-ray of the abdomen showed distension of the transverse colon only. Although there was no back pain he was thought to have had a retroperitoneal haemorrhage, and was treated conservatively with whole blood and platelets. Response was initially good, with the passage of a normal stool. Normal bowel sounds persisted despite the continuing abdominal distension. Rapid deterioration occurred again $24 \mathrm{hr}$ later with further upper abdominal distension but he now had clearly visible peristalsis with increased bowel sounds. Repeat abdominal X-rays showed an almost complete large bowel obstruction at the splenic flexure (Fig. 1). Despite transfusion his haemoglobin remained at pre-transfusion levels. He was considered to have continuing intra-abdominal haemorrhage either retroperitoneally or more likely submucosally, in view of the bowel obstruction, and laparotomy was performed.

Five and a half litres of blood were found in the peritoneal cavity together with a $6-\mathrm{cm}$ tear in the hilum of the spleen, furthermore the transverse colon was partially obstructed by extrinsic pressure from a splenic-hilar haematoma. Splenectomy was performed and adequate haemostasis achieved after transfusion of platelets. Removal of the haematoma resulted in collapse of the bowel, which was otherwise normal. Histology of the spleen showed a complete replacement of normal architecture by sheets of leukaemic cells.

\section{Subsequent events}

Postoperative recovery was stormy. He continued to bleed from the drain to the splenic bed which eventually became infected with Gram-negative organisms. Treatment with gentamicin and lincomycin resulted in a slow recovery. Chemotherapy for his leukaemia was resumed and he began to improve but unfortunately a Gram-negative septicaemia developed whilst at home for the week-end and the patient died within $1 \mathrm{hr}$ of readmission to hospital.

\section{Discussion}

Considering the vascularity of the spleen, its

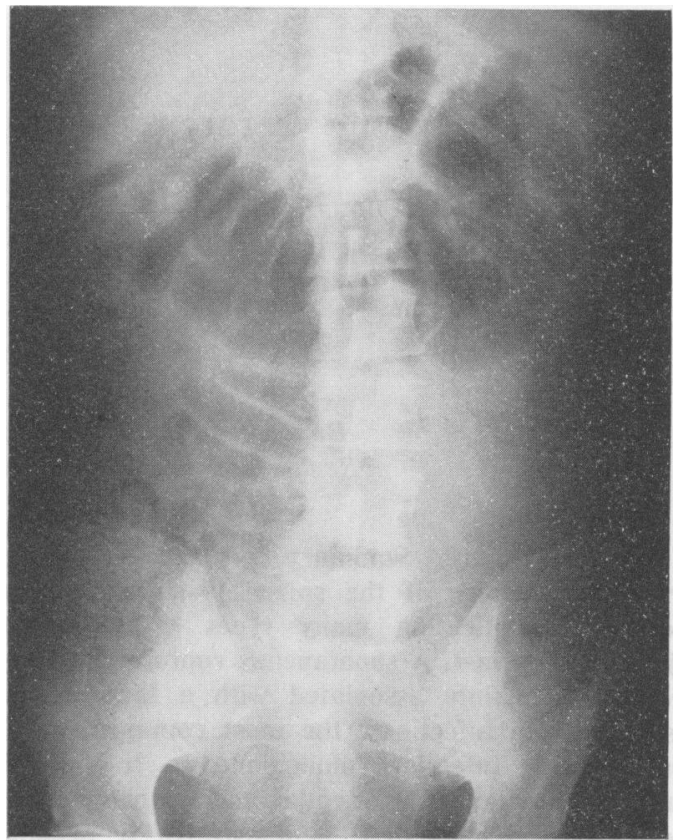

Fig. 1. Abdominal X-ray showing an almost complete large bowel obstruction to the splenic flexure.

relative lack of supportive tissue and the thin splenic capsule, it is surprising to find that spontaneous rupture of the organ is still an uncommon event. Spontaneous rupture of a normal spleen can be said to have occurred only if there has been no previous trauma, and examination of the organ, both macroscopically and microscopically, shows no abnormality apart from the laceration and haemorrhage. In a review in 1958 of seventy-one cases of spontaneous rupture, Orloff and Peskin considered that only twenty were truly spontaneous ruptures of a normal spleen, the rest occurred either as a result of trauma or in a pathological spleen, and a few had been inadequately documented. Naiberg, Sidlofsky and Chris (1965) added a further case conforming to the above criteria.

An enlarged spleen is removed from the protection of the diaphragm and rib case and it might be expected that spontaneous rupture would be more common in lymphomas and leukaemias but, although recorded, it is a rare event. In 1966, Stites and Ultmann, in reporting such a case in a patient with chronic lymphocytic leukaemia, could find only thirty-two other cases of ruptured spleen in leukaemia in the literature and it seemed that at most eight were spontaneous, it is interesting that only three cases of the thirty-two were in women. In the review referred to above, Orloff and Peskin did not state whether the ruptures of pathological spleens were of leukaemic or lymphomatous origin. Following the example 
of Walton (1963) this case should probably be described as a spontaneous rupture of a pathological spleen.

Although this patient was debilitated there was no history of trauma, indeed if the time of the first collapse is considered to be the time of rupture, then he was in bed. However, it may be that at the time of his third admission with faintness, the haemorrhage had already commenced and the collapse represented an extension of this process. Although pain was not a prominent feature of his illness, it did not occur at all until after his subsequent collapse.

At the outset, the clinical presentation was of retroperitoneal haemorrhage and in this respect was similar to the case recorded by Thompson in 1969. However, the development of large bowel obstruction is unusual. Ileus is a well recognized complication of retroperitoneal haemorrhage but bowel activity remained throughout this man's illness and before operation it appeared most likely that a submucosal haemorrhage had caused a local obstruction. At operation no such cause was found although there was a large volume of free blood in the peritoneal cavity and the obstruction appeared to be due to the large haematoma at the hilum of the spleen. For this reason the bowel was not opened. Furthermore, postoperatively there was no suggestion of recurrence of the obstruction.

This case demonstrates the varied clinical presentation with which a ruptured spleen may present and reminds one of its potential hazard in the management of the leukaemias and lymphomas.

\section{References}

NaiberG, D. Sidlofsky S. \& Chris, S.M. (1965) Spontaneous rupture of the spleen. Canadian Journal of Surgery, $8,96$.

Orloff, M.J. \& Peskin, G.W. (1948) Spontaneous rupture of the normal spleen-a surgical enigma. Internal Abstracts of Surgery, 106, 1.

Stites, T.B. \& UltmanN, J.E. (1966) Spontaneous rupture of the spleen in chronic lymphocytic leukaemia. Cancer, 19, 1587.

Thompson, W.H.F. (1969) Diffuse lymphocytic lymphoma with splenic rupture. Postgraduate Medical Journal, 45, 50.

Walton, T.J. (1963) Splenic rupture in infectious mononucleosis. Texas Journal of Medicine, 59, 333.

\title{
Hepatoma causing a massive tumour embolus
}

\author{
Y. F. DAJANI* \\ M.B., M.R.C.Path. \\ Dudley Road Hospital, Birmingham
}

\begin{abstract}
Summary
A case of hepatoma is reported, with a typical clinical setting but a most unusual and dramatic final episode. A massive tumour embolus weighing $30 \mathrm{~g}$ became arrested on the tricuspid valve, resulting in acute circulatory failure.

\section{Introduction}

Intravascular growth is an important feature of hepatoma (Edmondson, 1958). Although hepatoma has been reported to extend directly into the right atrium (Gregory, 1939), massive tumour embolism such as encountered in this case is not cited in the world literature.

\footnotetext{
* Present address: Department of Pathology, Faculty of Medicine, University of Jordan, Amman, Jordan.
}

\section{Case report}

A 66-year-old man was admitted with a history of recurrent leg swelling for the past 3 years. He had suffered increasing abdominal and leg swelling during the last 5 months with the development of icterus. He was found to have hepatosplenomegaly which had been noted 3 years earlier, during which time he was taking diuretics. Myocardial ischaemia with thyrotoxicosis had been treated 10 years earlier. $\mathrm{He}$ was a mild cigarette smoker and never a heavy drinker. Chest X-ray showed miliary shadowing.

Examination showed an ill-looking jaundiced man with generalized wasting. Ascites and massive hepatosplenomegaly were present. Liver cirrhosis, portal hypertension and hepatoma were diagnosed. He was treated with frusemide $40 \mathrm{mg}$ daily, and 